\title{
Exploring believable character animation based on principles of animation and acting
}

\author{
Nekhat Sultana, Dr Nico Meissner \& Dr Forest Lim \\ Yan Peng \\ Faculty of Creative Multimedia \\ Multimedia University \\ Cyberjaya, Selangor, Malaysia \\ nekhat.sultana@mmu.edu.my; \\ nico.meissner@mmu.edu.my; forest.lim@mmu.edu.my
}

\begin{abstract}
Character animation is a complex process, where the animator has to decide how a character on screen should move, emote and behave. It takes an animator years to polish his or her skills in order to deliver a believable animated character. Character animators, although given certain boundaries to work within, tend to be very subjective in their acting choices. This makes for a very complex and often complicated workflow to follow. Though approach to animating a character differs from one animator to another, there are two main frameworks utilized by many. The two frames works are - 12 Principles of Animation and Acting Principles. The latter set of principles is more current, developed only in $\mathbf{2 0 0 1}$ and thus lacks exposure among many novice animators. This papers reports the process combining the existing two frameworks in order to develop a more concise but simple workflow to assist the novice animator.
\end{abstract}

Keywords-12 Principles of Animation (PoA); Acting Principles; animation workflow

\section{INTRODUCTION}

In this research, we will explore the merging of the 12 Principles of Animation (PoA) (The principles are: Squash and Stretch, Anticipation, Exaggeration, Arc, Overlapping Action \& Follow Through, Secondary Animation Timing, Ease in \& Ease out / Slow in \& Slow out, Staging, Appeal, Solid Drawing, Personality and Straight ahead versus Pose to Pose Technique) with acting principles into one animation workflow that helps novice animators to create believable characters. Data for developing the animation workflow will be collected through literature as well as first hand experiences of professional animators manifested in weblogs, making-of videos, books and through interviews. The developed animation workflow will then be used to create a series of animated characters. The believability of these characters shall finally be evaluated by an audience in order to validate the developed workflow.

The animation workflow will allow novice animators to create work of a professional standard much earlier in their careers. This will benefit the Malaysian animation industry, which, though successful, still lacks the talent and manpower to keep up with national and international demand.

\section{BACKGROUND}

Animators are in constant pursuit of creating believability and bringing life to their characters (Jones, 1989). Thomas and Johnston (1994) wrote in The Illusion of Life that the characters they animated "appear to think and make decisions and act of their own volition... [creating] the illusion of life".

Animators therefore have to play the role of the puppeteer or the actor whereas the computer generated character is the puppet (Oore, Terzopoulous \& Geoffrey, 2002).

The general method followed is usually by starting with rough sketches on paper or digital sketches in the computer. Once the key poses refined, the animator will then use these as references for setting up the keyframes in a 3D software (Lasseter, 1994).

When developing the key poses, the 'personality' of a character is the motivator of its actions. A character's movement should be portrayed as the result of its thought process (Bates, 1994). This makes the character appear alive and almost realistic in terms of behavior and creates an intimate relationship between the audience and the character. This relationship generates the empathy needed by the audience to connect with the character on screen (Lasseter, 1987 and Thomas \& Johnston, 1994).

Experienced animators follow two main concepts when developing the 'personality' of a new character.

\section{TWO CONCEPTS TO THIS RESEARCH}

\section{A. Acting Principles}

Stanislavski was the first to promote representational forms of acting (Kundert-Gibbs, 2009). Everyday communication becomes a reference point for actors. They observe people, situations and occurrences in reallife settings and use these experiences to create their characters (Naremore, 1990). As a consequence, each character is unique since no one person moves, reacts or behaves the same. An animator has to approach acting in a similar manner. Bjorklund (2009) referred to acting principles developed by acting coach Ed Hooks (2005) when he was training animators. According to Bjorklund and Hooks, these concepts are important and need to be understood by an animator in order for them to develop a believable character. 
In his pursuit to create a comprehensive structure to train animators as actors, Ed Hooks developed guidelines to help animators bridge the gap between drawing and bringing life to an animated character. These guidelines are known as the Acting Principles. Although Hook claims there is no 'right' way to deliver a performance, the guidelines below are of help when deciding on 'acting choice'.

A scene is a negotiation : Each scene should contain some form of conflict or obstacle. This keeps the story and scene interesting. The character has a purpose in each scene. There are only three types of conflicts in storytelling : [1] conflict with self; [2] conflict with the situation; [3] conflict with another character.

Thinking leads to conclusion; emotion tends to lead to action : Walt Disney has always maintained the character should lead with the mind, the thinking process should be shown (Hooks, (2005), Thomas \& Johnston, (1994). All actions should begin with a thought, which translate into a particular emotion, and then the body responds by moving accordingly.

Acting is Reacting. Acting is doing : Acting can mean a character is doing something OR reacting to something. A character by right is always reacting to something, be it another character, to his/her environment, an obstacle, etc.

Character needs to have an objective : Referencing to theater acting concept, in an animation piece, audience needs to be able to understand what the character is doing at all times. He should always be in pursuit of an objective. A character needs to move with a sense of purpose.

Character should play an action until something happens to make him play a different action : An animated character should always be seen to be doing something, or it will look dead very quickly. Even breathing has to be seen. There can be many factors, be internal (thoughts) or external (actions) that may lead the character to do something. The concept of a bead necklace was introduced by Stanilavsky as a metaphor for an action by an actor. String of beads is like a sequence of continuous action. There is no break between the beds, and therefore there really is no pause in between actions of a character.

All action begins with movement : In animation, something as miniscule as the thinking process has to be shown in some manner - usually with the rolling of the eyeball. When the thinking process is completed the character will have an answer which will lead him to the movement or action he/she needs to do. According to Hooks, action without movement is impossible.

Empathy is the key to connecting with audience : Connecting with the audience is important in order for the audience to have empathy with the character. This could be done by making sure the character is able to relate to the audience on a personal level. Development of personality of the character here is crucial in order to create a character that is able to accessible to the audience, in order to build a sense of empathy for the character.

\section{B. Principles of Animation (PoA)}

Movement of a character in animation is very crucial. All movements should be fluid and look as believable as possible.

In the 1920s, Disney's animators developed a set of practices that became known as the Principles of Animation (PoA). These guidelines have been used to date (Thomas \& Johnston, 1994). The principles are: Squash and Stretch, Anticipation, Exaggeration, Arc, Overlapping Action \& Follow Through, Secondary Action, Timing, Ease in \& Ease out / Slow in \& Slow out, Staging, Appeal, Solid Drawing, Personality and Straight ahead versus Pose to Pose Technique.

Squash \& Stretch: This particular principles looks at the displacement of mass of the moving object. This change in shape of an animated object usually happens when it comes into contact with another object or with a surface. The main thing to keep in mind is that the overall volume or mass of the object does not change, although the shape might.

Anticipation : Every action has a visual cue that informs the audience of the action the character is about to perform. It is important to design the anticipation so the audience is able to follow the action and story.

Exaggeration : This principle looks at magnifying or reducing a particular action or emotion to a certain extreme. Exaggeration tends to work well with several other principles of animation such as Squash \& Stretch, Timing, and Slow-in \& Slow-out. Exaggeration can also be applied to the editing, or cinematography in order to increase the intensity of a particular scene or shot.

$\underline{\operatorname{Arc}}:$ All objects animated tend to move in a fluid arclike motion. All organic beings have this natural motion path, unlike mechanical objects or characters, which may have a more straight and rigid movement.

Overlapping Action \& Follow Through : This particular principle adds layers to a particular action of a character. It makes the movement much more believable. 
Examples of this principle are elements such as clothing, hair, tail, floppy ears - these elements will move or react to the movement of the main body when for example, the character walks, jumps or hops. When a character stops his/her main action, the overlapping action will eventually come to a stop as well, just not exactly at the same time. A good example is of a girl wearing a long flowing skirt. If she stops walking, the skirt will eventually stop sashaying a few seconds later.

Secondary animation : Secondary animations are smaller bits of action that are designed to complement the main action done by a character. A character waiting for the bus might do certain actions such as tap his feet, or keep looking at his wristwatch impatiently. This also gives the audience a peek into the character's personality.

Timing : Timing is probably one of the more involved principles here, as it also works in tandem with other principles of animation to relay believable animation. Timing is crucial in dictating emotions of the character. Sad characters tend to move slowly, happy characters tend to have a faster pace. Angry characters tend to move really fast, if the character is aggressive. Passive anger may be shown as extremely slow-motion before everything blows up (Kerlow, 2009).

Ease in/ Ease out : Also known as Slow-in/ Slow-out, this principle is applied at the beginning of action or at the end of an action. The principle can work hand in hand with other principles to create anticipation, or to execute a follow through element of a character.

Staging : This principle derives from methods during theater plays. The whole purpose of design the staging of a scene is to make sure an idea is put across to the audience, and that the idea is clear in its intention. The action of the character must be clearly communicated to the audience.

Appeal : This principle looks at developing a wellrounded character be it a positive or a negative characters. All characters in a story need to have appeal in order to connect with the audience, or the audience wouldn't care about them.

Solid Drawing : This principle was developed specifically for hand-drawn animation, but applies also to 3D animation in principle. The whole purpose of this principles is to make sure characters have clear delineation of shape in order to be sure there is clarity when being animated and shown to the audience. A clear silhouette is a good test of solid drawing. If an animator can see clearly what the character is doing (or not doing) in the silhouette study of the character, then it is likely the audience will be clear as well (Kerlow, 2009).

Straight ahead vs Pose to Pose Technique : This particular principle refers to method of animating a character. Pose-to-pose was popular method of animating when the industry was still based on 2D animation. This method was quite optimum in getting animation done quickly, as main animator only worked on timing and the key poses in a scene or shot. The rest of the in-between drawings and spacing are completed by assistant animators or in-betweeners. Sometimes characters have a more spontaneous, erratic or organic move, such as a frantic run, or a floating balloon. In this case, straight-ahead animation is more appropriate in capturing the movement. The animator will therefore draw every frame until he/she captures the motion.

Personality : Although not actually a principles, this particular attribute was added by Lasseter for a paper he presented in 1987 (Lasseter, 1987). Lasseter omitted out Solid Drawing from his list of 12 Principles of Animation, and added Personality as a binding agent that motivates the usage of all the other principles. Having a clear personality of the character is important in order to decide how the 11 principles are applied. It will give indication to what kind of emotions the character has and therefore how he/she/it would react in any given scenario.

\section{RESEARCH PROBLEM}

Novice animators are so accustomed to the 12 Principles of Animation that they often cut out other aspects of references when it comes to developing a believable character (Hosea, 2010). Rather than the PoA being a first step in the exploration of motion and emotion, they have simply been reduced to a calculative simulation of life-like behavior. Novice animators will memorize and regurgitate these principles in their animation, referencing to other animations, rather than observing reality. This has in turn given their works a very shallow and skewed interpretation of the 12 Principles of Animation and limits their ability to observe real life around them. They are thus unable to understand how appropriate implementations are to be carried out in order to deliver a believable character animation. Consequently, novice animators tend to produce animations that lack believability (see also Bishko, 2007).

On an academic level, there have been many studies on human behavior in animation. But these are primarily targeting emotional facial expressions (for instance Sloan, Cook, Robinson, 2009) or focus on static images, such as photographs, drawings or static computer generated images (for instance Bassili, 1979) rather than moving characters.

\section{RESEARCH OBJECTIVES}

The study will take the 12 PoA as a conceptual starting point and merge them with acting principles that are applied by contemporary experienced animators when they develop a new animated character. Overall objective of the research is : 
- to identify the various frameworks available as practiced by professional animators.

- $\quad$ to study the acting tools available, and applied by professional animators in delivering a believable animated character.

- to develop an optimized framework based on acting tools to be applied into a character animation pipeline.

\section{RESEARCH QUESTION}

Many beginner animators tend to rely heavily on the 12 PoAs as the main source of understanding character movement when animating, thus creating an animated character that is lacking in believability (Bishko, 2007 \& Bird, 2003). This problem also limits the new animator's ability to observe from their surroundings, and how to apply any observation if any, into their animation pipeline (Bishko, 2007 \& Hosea, 2010). As acting skills have been known to be a useful tool in understanding characters (Thomas \& Johnston, et all), Bishko has suggested that new animators could refer to acting tools in order to understand motion and movement.

Research Question 01 : What are other frameworks available for a new animator to work with, in order to deliver a believable animated character?

Research Question 02 : What are the acting tools required for an animator?

Research Question 03 : How can the acting tools be applied into a character animation pipeline?

\section{DESCRIPTION OF METHODOLOGY}

\section{A. Exploration Through Conceptualization}

Based on data collected from literature and firsthand accounts of professional animators (the latter manifested in books, weblogs, making-of video clips as well as personal interviews), an animation workflow will be developed that lists and explains the all the steps professional animators take when developing a new character. The workflow will be developed in such a way that it can be understood and used by animation students, and other novice animators.

\section{B. Feedback by Industry Practitioner}

Using the conceptual framework as a starting point, the researcher will present the framework to industry practitioners for feedback. This will be done through open-ended interviews, focusing on the industry practices, which may differ from one company to another.

\section{Verification}

The data from the interviews will be analyzed, and may be absorbed into the conceptual frame to make it more concise and reflect the industry practice in a more accurate manner. ?

\section{DISCUSSION}

There are many books that attempt to teach novice animators how to animate a character. The vast majority of these exclusively focus on Disney's concept of the 12 PoA. Professional animators, however, work differently. They also use principles known from acting. This research project wants to develop and test a new approach, a new workflow that helps novice animators to create more believable characters.

This new workflow, if followed by novice animators, is likely to rapidly advance these animators' ability to create believable characters of a professional standard. This, in turn, will help the pool of talent in the local Malaysian animation industry to generate more contractual work for overseas, often Hollywood, studios as well as enable them to develop a unique, Malaysian voice in the animation industry.

The primary objective of the study is to develop and test an animation workflow that helps new animators deliver a believable animated character through the application of acting principles and the 12 Principles of Animation.

Based on this merging of PoA and acting principles, we will explore a new animation workflow that will allow novice animators to understand the overall working process and thinking used by professional animators when animating a character. This new animation workflow will help novice animators to created more believable characters by no longer exclusively relying on the rather archaic concept of Disney's PoA but combining it with principles that are used by today's leading animators. This, in turn, will help novice animators to created more believable characters by no longer exclusively relying on the rather archaic concept of Disney's PoA but combining it with principles that are used by today's leading animators. This, in turn, will allow new animators to create believable characters of a professional standard much earlier in their careers.

[1] Jones, C. (1989). The Life and Times of an Animated Cartoonist. New York: Farrar, Straus \& Giroux. 
[2] Thomos, F., Johnston O. (1994). The Illusion of Life: Disney Animation (2nd ed.). New York: Hyperion.

[3] Oore, S; Terzopoulos, D; Hinton, G. (2002). A Desktop Input Device and Interface for Interactive $3 D$ Character Animation. In Proc. Graphics Interface, 133-140.

[4] Lasseter, J. (1987). Principles of Traditional Animation Applied to 3D Computer Animation. Computer Graphics, 21(4), 35-44.

[5] Bates, J. (1994). The role of emotion in believable agents. Communications of the ACM, 37(7), 122-125

[6] Kundert-Gibbs, J., Kundert-Gibbs, K. (2009). Action! Acting Lessons for CG Animators. Singapore: Wiley Publishing

[7] Naremore, J. (1990). Acting in the cinema. Los Angeles: University of California Press.

[8] Björklund, N. (2009). Acting in Animation. Department of Mathematics, Natural and Computer Science.

[9] Hooks, E. (2005). Acting in Animation, A Look at 12 Films. Portsmouth, New Hampshire: Heinemann

[10] Kerlow, I. (2009). The Art of 3D Computer Animation and Effects ( $4^{\text {th }}$ Edition $)$. John Wiley \& Sons, Inc., Hoboken, New Jersey.

[11] Hosea, B. (2010). Drawing Animation. Animation: An Interdisciplinary Journal, 5(3), 353-367.

[12] Bishko, L. ( 2007). The Uses and Abuses of Cartoon Style in Animation. Animation Studies 2, URL (consulted February 2013): http://journal.animationstudies.org/category/volume-2/lesliebishko-the-uses-and-abuses-of-cartoon-style-in-animation/

[13] Sloan, R.J.S., Cook, M., Robinson, B. (2009). Considerations for believable emotional facial expression animation. Second International Conference in Visualisation, Barcelona, 15-17 July 2009. pp.61-66. 